\title{
EDITORIAL
}

\section{DIABETES, UNA MIRADA GLOBAL}

Haber participado en la coordinación del XIX Congreso Argentino de Diabetes fue un placer enorme y una experiencia sumamente enriquecedora. El Programa Científico -cuyo desarrollo llevó casi un año- intentó abarcar todo el espectro de temas relacionados con la diabetes, yendo desde lo molecular hasta lo estrictamente tecnológico, sin descuidar por supuesto los aspectos clínicos de esta patología. Teniendo en cuenta estas temáticas, se pensó en un lema que reflejara este espíritu, de allí que el mismo fuera "Diabetes, un debate constante".

Además de los temas que "clásicamente" suscitan discusión y que por su frecuencia de presentación y/o su relevancia diagnóstica o terapéutica son parte rutinaria de nuestra actividad (tales como insulinización oportuna en personas con diabetes tipo 2 , incretinas, nuevos análogos de insulina, inhibidores de SGLT2, aspectos genéticos, entre otros) se decidió, en esta oportunidad, incluir en el programa temáticas relacionadas con una mirada más global de la diabetes, es por ello que se eligieron como invitados extranjeros a profesionales como el Dr. Guillermo Umpiérrez, quien en su magistral conferencia de cierre "Visión crítica del tratamiento de la diabetes tipo 2" realizó un resumen de parte de lo interactuado durante los tres días que duró el Congreso.

Además hubo una importante actividad pre-congreso desarrollada por los integrantes del Capítulo Atlántico, cuyo eje central fue la "Atención primaria de la salud en diabetes y embarazo." A la misma asistieron miembros del equipo de salud quienes participaron de manera entusiasta en los talleres presentados en la misma.

Por primera vez se llevó a cabo en paralelo al Congreso y de manera conjunta con el Ministerio de Deporte de la Nación, a través de la gestión de la
Dra. Silvia Gorbán de Lapertosa, una actividad que incluyó a jóvenes con diabetes (a la cual asistieron aproximadamente 70 de distintos lugares del país); el encuentro fue verdaderamente enriquecedor no sólo para ellos, sino también para los profesionales encargados de su concreción.

De la evaluación del número de inscriptos que afortunadamente crece constantemente, pasando de 2.751 en el año 2012 a casi 3.100 en este último año, surge muy claramente el creciente interés por el Congreso Argentino de Diabetes, no sólo por parte de los médicos especializados en el área, sino por profesionales de diversas especialidades como la Clínica, Cardiología o Nefrología, entre otras.

Agradecemos a todos los socios que participaron activamente, y asistieron a gran parte de los simposios y conferencias, en los que se fomentaron el debate y la discusión y en los cuales no faltaron preguntas enriquecedoras. Cabe destacar que los salones han estado completos en su capacidad, a pesar de los hermosos días de sol, que podrían haber tentado a varios a concurrir a la playa.

Agradecemos a la industria farmacéutica que nos apoyó para que el Congreso pudiera realizarse y que, con gran esfuerzo, organizó y garantizó la llegada y partida de sus profesionales invitados en los horarios adecuados.

Las actividades finalizan y luego, necesariamente, debemos hacer un análisis de todos sus aspectos. Nos sentimos satisfechos y orgullosos de este Congreso por la variedad y riqueza de temas de alguna manera no convencionales y por el clima de camaradería con el que transcurrieron.

Sin lugar a dudas este XIX Congreso Argentino de Diabetes ha cumplido el objetivo: ser un punto de encuentro de quienes formamos parte de esta Sociedad para discutir y aprender.

Dra. María Cristina Faingold Coordinadora del Comité Científico del XIX Congreso Argentino de Diabetes 